\title{
Chromosomal-level genome assembly of silver sillago (Sillago sihama)
}

\author{
Changxu Tian ${ }^{1}$, Xinghua Lin ${ }^{1}$, Yang Huang ${ }^{1}$, Huapu Chen ${ }^{1}$, Dongneng Jiang ${ }^{1}$, Hongjuan \\ $\mathrm{Shi}^{1}$, Siping Deng ${ }^{1}$, Tianli Wu ${ }^{1}$, Yulei Zhang ${ }^{1}$, Mouyan Jiang ${ }^{1}$, Tao Du ${ }^{1}$, Chunhua Zhu ${ }^{1}$, \\ and Guangli $\mathrm{Li}^{1}$
}

${ }^{1}$ Guangdong Ocean University

October 6, 2020

\begin{abstract}
Silver sillago, Sillago sihama is a member of the family Sillaginidae and found in all Chinese inshore waters. It is an emerging commercial marine aquaculture species in China. In this study, high-quality chromosome-level reference genome of S. sihama was first constructed using PacBio Sequel sequencing and high-throughput chromosome conformation capture (Hi-C) technique. A total of $66.16 \mathrm{~Gb}$ clean reads were generated by PacBio sequencing platforms. The genome-scale was $521.63 \mathrm{Mb}$ with 556 contigs, and $13.54 \mathrm{Mb}$ of contig N50 length. Additionally, Hi-C scaffolding of the genome resulted in 24 chromosomes containing $96.93 \%$ of the total assembled sequences. A total of 23,959 protein-coding genes were predicted in the genome, and $96.51 \%$ of the genes were functionally annotated in public databases. A total of $71.86 \mathrm{Mb}$ repetitive elements were detected, accounting for $13.78 \%$ of the genome. The phylogenetic relationships of silver sillago with other teleosts showed that silver sillago was separated from the common ancestor of S. sinica about 7.92 million years ago. Comparative genomic analysis of silver sillago with other teleosts showed that 45 unique and 100 expansion gene families were identified in silver sillago. Expansion gene families were involved in immune and olfactory receptors. In this study, the genomic resources provide valuable reference genomes for functional genomics research of silver sillago.
\end{abstract}

\section{Hosted file}

Main Document.pdf available at https://authorea.com/users/311465/articles/485117-chromosomallevel-genome-assembly-of-silver-sillago-sillago-sihama 